\title{
Design, Synthesis and Structural Confirmation of a Series of 2-(Thiophen-2-yl)- 4H-chromen-3-yl-sulfonate Derivatives and Preliminary Investigation of Their Antioxidant and Anticancer Potentials
}

\author{
Jialin Zang, ${ }^{a, b}$ Ming Bu, Jifang Yang, ${ }^{c}$ Lu Han ${ }^{c}$ and Zhen Lv ${ }^{\circledR *, c}$ \\ ${ }^{a}$ The Second Affiliated Hospital of Qiqihar Medical University, 161006 Qiqihar, China \\ ${ }^{b}$ The First Affiliated Hospital of Jiamusi University, 154007 Jiamusi, China \\ ${ }^{c}$ College of Pharmacy, Qiqihar Medical University, 161006 Qiqihar, China
}

\begin{abstract}
A series of novel 2-(thiophen-2-yl)-4H-chromen-3-yl-sulfonate derivatives (4a-4n) were synthesized and investigated for their in vitro free radical scavenging potential as well as cytotoxic efficacies against selected cancer cell lines. The cytotoxicity of the $4 H$-chromene derivatives (4a-4n) was evaluated according to three human cancer cell lines (HepG2, A549, HeLa) by utilizing a 3-(4,5-dimethylthiazol-2-yl)-2,5-diphenyltetrazolium bromide (MTT) assay. Accordingly, part of the results exhibited better cytotoxic activities than that of the positive controls ( $4 \mathrm{H}$-chromen-4-one and apigenin). Among them, compounds $\mathbf{4 c - 4 g}$ exhibited better training to the positive control against the three human cancer cell lines (half maximal inhibitory concentration $\left(\mathrm{IC}_{50}\right)=3.87 \pm 0.12$ to $21.38 \pm 0.52 \mu \mathrm{M})$. Moreover, the extract of the $4 H$-chromene derivatives $(\mathbf{4 a}-\mathbf{4 n})$ showed better activity against 2,2-diphenyl-1-picrylhydrazyl (DPPH) and 2,2'-azino-bis-3-ethylbenzothiazoline6-sulfonic acid (ABTS) in antioxidant assays compared to that of the positive control ascorbic acid $\left(\mathrm{IC}_{50}=12.72 \pm 0.27,5.09 \pm 0.21 \mu \mathrm{g} \mathrm{mL}-1\right)$. Thus, it can be confirmed from the bioassay results that the overall structural design, as well as proper substitution, is crucial in delivering anticipated biological effects. In this regard, spectroscopic techniques such as ${ }^{1} \mathrm{H}$ nuclear magnetic resonance (NMR), ${ }^{13} \mathrm{C}$ NMR, and high-resolution mass spectrometry (HRMS) were also carried out to confirm the final structures.
\end{abstract}

Keywords: $4 H$-chromen, structural confirmation, cytotoxicity, antioxidant activity

\section{Introduction}

Drug discovery based on natural products is the main way to develop drugs for the treatment of various diseases, especially in the treatment of cancer. ${ }^{1} 4 H$-Chromene, a heterocyclic system consisting of a fusion of a benzene ring and a pyran ring, is an essential structural component of natural compounds, like flavonoids, coumarins, and tocopherols. Various natural and synthetic derivatives of chromenes possess critical biological and pharmacological applications, such as anti-inflammatory, antiproliferative, antioxidant, antimicrobial, anti-HIV, anti-tubercular, antitumor, cytotoxic, antidyslipidemic, antileishmanial, anti-inflammatory, anti-Helicobacter pylori, and TNF- $\alpha$ inhibitor agents. ${ }^{2-7}$ Nowadays, most $4 \mathrm{H}$-chromene derivatives are applied by modifying the benzopyran and side chains.

*e-mail: pangmax27@163.com
Due to its unique and attractive chemical structure, it is continually being explored by scientists. The halogen, heteroatom, amino acid, and amide was also introduced into the system with the hope of discovering novel chemical entities with the potential to be future therapies, bringing about notable modifications of its biological and pharmacological activities. ${ }^{8}$

Numerous $4 H$-chromene derivatives have been found in nature, and as essential active lead compounds in drug discovery, their modification has been explored in order to obtain more active drugs. Almost every group of $4 H$-chromene derivatives has antioxidant effects. According to reports, ${ }^{9-11}$ flavonoids and catechins seem to be the most powerful $4 \mathrm{H}$-chromene derivatives used to protect the body from the effects of active oxygen. The cells and tissues of the body were continuously threatened by causing free radicals and reactive oxygen species, which were produced in the process of normal oxygen metabolism or induced by 
<smiles></smiles>

Quercetin<smiles>O=c1c(O)c(-c2ccc(O)cc2)oc2cc(O)cc(O)c12</smiles>

Kaempferol<smiles>O=c1c(O)c(-c2ccc(O)c(O)c2)oc2cc(O)ccc12</smiles>

Fisetin

Figure 1. Representative compounds.

exogenous damage. Among them, the most representative compounds are quercetin, kaempferol ${ }^{12}$ and fisetin ${ }^{13}$ (Figure 1). Numerous $4 H$-chromene derivatives with various heterocycle structural motifs have been recently reported and evaluated as antitumor and antioxidant agents. ${ }^{14-19}$

Fentanyl and sufentanil are common opioid analgesics (Figure 2). Better antipyretic and analgesic effects are demonstrated by sufentanil, because of thiophene. Simultaneously, compared to fentanyl, sufentanil demonstrated comparable analgesic efficacy and safety with less analgesic consumption (under a potency ratio of 1:5) in intravenous patient-controlled analgesia (IV-PCA) following total laparoscopic hysterectomy. ${ }^{20}$

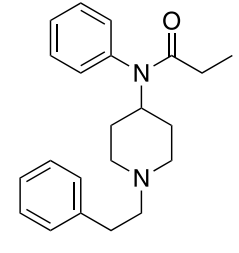

Fentanyl

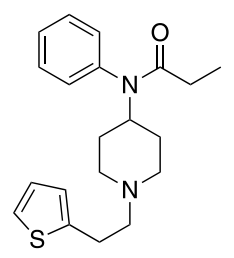

Sufentanil
Figure 2. Molecular structures of fentanyl and sufentanil.

Studies ${ }^{21}$ have shown that these $4 H$-chromene derivatives, such as epimedoside, lignan, baohuoside, hypericin, and chaohuoside B, have both scavenging effects on 2,2-diphenyl1-picrylhydrazyl (DPPH) radicals and inhibiting effects on breast cancer cells (MCF-7) and liver cancer cells (HepG 2), but not the stronger the scavenging ability of free radicals, the stronger their ability to inhibit tumor cell proliferation. However, as far as other polyphenolic compounds are concerned, some studies have shown that the better the antioxidant activity is, the better its anti-tumor ability is, but there is also literature showing that antioxidants do not have tumor-inhibiting ability. ${ }^{22,23}$

Arylsulfonyl belong to an important class of organic functional group, which is widely used in medicinal chemistry. Hydantoin-based derivatives were reported to significantly inhibit ovarian and renal cancer cells. ${ }^{24}$ Additionally, a dimethoxyimidazolidinone core with one or two arylsulfonyl groups possessed good inhibitory effects on lung and renal cancer cells and demonstrated potential as carbonic anhydrase inhibitors ${ }^{25}$ (Figure 3a). Moreover, compounds bearing arylsulfonyl moiety may show selective COX-2 inhibition activity ${ }^{26}$ (Figure $3 b$ ). In the present study, new $4 H$-chromene derivatives were synthesized. The modification was made through variations in the thiophene groups and arylsulfonyl moieties. The synthesized compounds were evaluated for their antioxidant and cytotoxic activities.
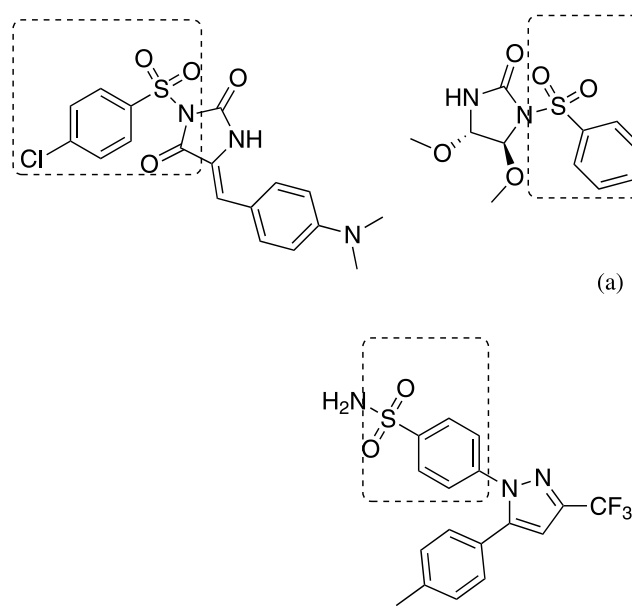

Celecoxib

(a)
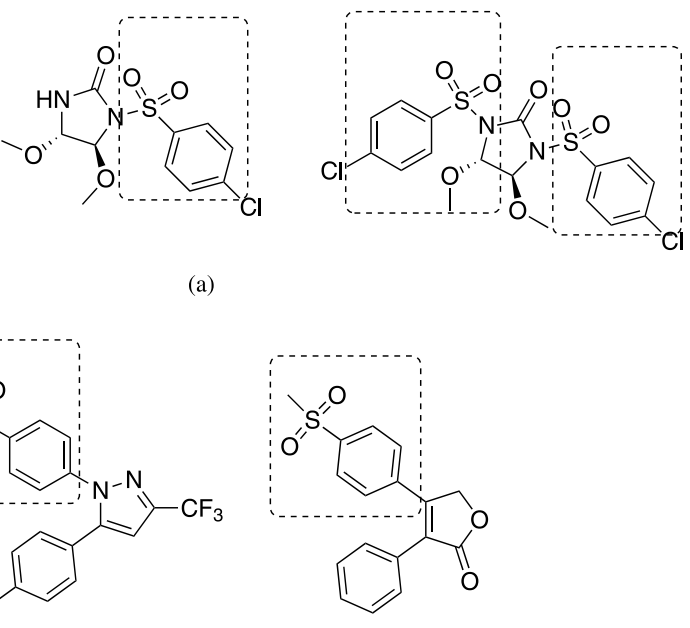

Rofecoxib

(b)

Figure 3. Molecular structures of arylsulfonyl derivatives. 


\section{Experimental}

Chemsitry

All reagents and solvents were purchased from commercial sources (Energy, Shanghai, China) and used without further purification. All reactions dealing with air and moisture-sensitive compounds were carried out under an atmosphere of argon using flame-dried glassware and standard syringe techniques. Unless otherwise noted, all retail reagents and solvents were obtained from the commercial provider and used without further purification. ${ }^{1} \mathrm{H}$ and ${ }^{13} \mathrm{C}$ nuclear magnetic resonance (NMR) spectra were recorded on Bruker $400 \mathrm{MHz}$ spectrometers (Berlin, Germany). Chemical shifts were reported relative to internal tetramethylsilane $(\delta 0.00 \mathrm{ppm})$ or dimethyl sulfoxide (DMSO- $\left.d_{6}, \delta 7.26 \mathrm{ppm}\right)$ for ${ }^{1} \mathrm{H}$ and DMSO- $d_{6}(\delta 77.00 \mathrm{ppm})$ for ${ }^{13} \mathrm{C}$. High-resolution mass spectrometry (HRMS) was recorded on Agilent 1100 LC/QTOF spectrometer (San Francisco, USA).

(E)-1-(2-Hydroxyphenyl)-3-(thiophen-2-yl)prop-2-en-1-one $(2 a-2 c)$

A mixture of thiophen-2-carbaldehyde (1a-1c, 2 mmol) and $o$-hydroxyacetophenone $(2.4 \mathrm{mmol})$ were dissolved in $40 \mathrm{~mL}$ methanol and methanolic potassium hydroxide solution ( $1 \mathrm{~mL}, 10 \mathrm{mmol} \%)$. The temperature was raised to $80{ }^{\circ} \mathrm{C}$ and refluxed for $4 \mathrm{~h}$. The reaction was cooled to room temperature and adjusted to $\mathrm{pH} 3-4$ with $1 \mathrm{M} \mathrm{HCl}$. Then, it was filtered and washed with petroleum ether to give yellow solid (2a-2c).

\section{$2 a$}

Yellow solid; 92\%; ${ }^{1} \mathrm{H}$ NMR (400 MHz, DMSO- $d_{6}$ ) $\delta 12.38$ (1H, s, H12), 8.13 (1H, dd, J 8.2, 1.6 Hz, H9), 8.01 (1H, d, J 15.2 Hz, H3), 7.87-7.82 (1H, m, H8), 7.76-7.65 (2H, m, H13, H15), 7.56 (1H, ddd, J 8.5, 7.2, $1.6 \mathrm{~Hz}, \mathrm{H} 5)$, 7.23 (1H, dd, J 5.1, 3.7 Hz, H14), 7.03-6.94 (2H, m, H4, H6); ${ }^{13} \mathrm{C}$ NMR (100 MHz, DMSO- $\left.d_{6}\right) \delta 193.6$ (C7), 163.7 (C1), 137.6 (C10), 136.4 (C9), 133.8 (C5), 131.6 (C13), 131.0 (C3), 129.3 (C15), 126.7 (C14), 121.0 (C2), 120.9 (C8), 119.5 (C4), 118.2 (C6).

\section{2b}

Yellow solid; 91\%; ${ }^{1} \mathrm{H}$ NMR (400 MHz, DMSO- $\left.d_{6}\right)$ $\delta 8.53$ (1H, s, H12), 7.88 (1H, d, J 15.0 Hz, H9), $7.66(1 \mathrm{H}$, dd, J 7.4, 2.0 Hz, H3), 7.59-7.50 (2H, m, H5, H13), 7.28 (1H, d, J $15.0 \mathrm{~Hz}, \mathrm{H} 8), 7.07$ (1H, dd, J 7.5, 2.0 Hz, H6), 7.04-6.98 (2H, m, H4, H14), 2.54 (3H, s, H17); ${ }^{13} \mathrm{C}$ NMR (100 MHz, DMSO- $d_{6}$ ) $\delta 193.6(\mathrm{C} 7), 163.7$ (C1), 145.8
(C15), 140.1 (C10), 135.9 (C5), 135.4 (C9), 131.4 (C13), 130.0 (C3), 127.6 (C14), 121.7 (C2), 121.1 (C8), 119.9 (C4), 118.7 (C6), 15.8 (C17).

2c

Yellow solid; 95\%; ${ }^{1} \mathrm{H}$ NMR (400 MHz, DMSO- $d_{6}$ ) $\delta 7.92$ (1H, d, J 15.0 Hz, H9), 7.62-7.51 (3H, m, H3, H5, H9), 7.29-7.19 (2H, m, H13, H14), 7.11 (1H, dd, J 7.5, $2.0 \mathrm{~Hz}, \mathrm{H} 6), 7.01(1 \mathrm{H}, \mathrm{td}, J 7.5,2.0 \mathrm{~Hz}, \mathrm{H} 4) ;{ }^{13} \mathrm{C} \mathrm{NMR}$ (100 MHz, DMSO- $d_{6}$ ) $\delta 193.6$ (C7), 163.7 (C1), 142.3 (C10), 135.9 (C5), 135.4 (C9), 130.6 (C14), 130.0 (C3), 126.7 (C13), 121.7 (C2), 121.1 (C8), 119.9 (C4), 118.7 (C6), 116.1 (C15).

3-Hydroxy-2-(thiophen-2-yl)-4H-chromen-4-ones (3a-3c)

Compounds 2a-2c (2 mmol) and $\mathrm{KOH} / \mathrm{MeOH}(5 \mathrm{~mL}$, $10 \mathrm{mmol} \%)$ were solubilized in $\mathrm{MeOH}(20 \mathrm{~mL})$. The temperature was reduced to $0{ }^{\circ} \mathrm{C}$ and refluxed for $0.5 \mathrm{~h}$. Then, $8 \mathrm{~mL} \mathrm{H}_{2} \mathrm{O}_{2}$ was dropped into the mixture in the meanwhile the solution switched from red to yellow. The yellow precipitate was collected and washed with water and petroleum ether for twice to give intermediates $\mathbf{3 a - 3 c}$.

$3 a$

Yellow solid; 94\%; ${ }^{1} \mathrm{H}$ NMR (400 MHz, DMSO- $d_{6}$ ) $\delta 10.29$ (1H, s, H16), 8.11 (1H, d, J 7.9 Hz, H5), $7.98(1 \mathrm{H}$, d, J $3.6 \mathrm{~Hz}, \mathrm{H} 7), 7.92(1 \mathrm{H}, \mathrm{d}, J 4.9 \mathrm{~Hz}, \mathrm{H} 8), 7.80(1 \mathrm{H}, \mathrm{t}$, $J 7.7 \mathrm{~Hz}, \mathrm{H} 14), 7.73$ (1H, d, J $8.3 \mathrm{~Hz}, \mathrm{H} 6), 7.47$ (1H, t, $J 7.4 \mathrm{~Hz}, \mathrm{H} 12), 7.31$ (1H, t, J $4.4 \mathrm{~Hz}, \mathrm{H} 13) ;{ }^{13} \mathrm{C}$ NMR (100 MHz, DMSO- $d_{6}$ ) $\delta 177.7$ (C4), 153.7 (C9), 148.8 (C2), 143.3 (C11), 135.9 (C3), 131.5 (C7), 126.8 (C14), 126.0 (C13), 125.2 (C12), 123.0 (C5), 122.4 (C6), 122.1 (C10), $118.2(\mathrm{C} 8)$

3b

Yellow solid; 90\%; ${ }^{1} \mathrm{H}$ NMR (400 MHz, DMSO- $d_{6}$ ) $\delta 8.19(1 \mathrm{H}, \mathrm{dd}, J 7.5,2.0 \mathrm{~Hz}, \mathrm{H} 5), 7.87$ (1H, dd, J 7.5, $2.0 \mathrm{~Hz}, \mathrm{H} 7), 7.66(1 \mathrm{H}, \mathrm{dd}, J 7.5,2.0 \mathrm{~Hz}, \mathrm{H} 8), 7.55$ (1H, dd, $J$ 7.4, $2.0 \mathrm{~Hz}, \mathrm{H6}), 7.23$ (1H, d, J 7.5 Hz, H12), $6.91(1 \mathrm{H}$, d, J 7.5 Hz, H13), 6.68 (1H, s, H16), 2.47 (3H, s, H17); ${ }^{13} \mathrm{C} \mathrm{NMR}\left(100 \mathrm{MHz}, \mathrm{DMSO}-d_{6}\right) \delta 173.9$ (C4), 155.2 (C9), 152.0 (C2), 145.3 (C14), 136.7 (C11), 135.4 (C3), 134.5 (C7), 129.4 (C12), 126.2 (C13), 125.1 (C5), 124.5 (C6), 121.5 (C10), 118.0 (C8), 16.0 (C17).

3c

Yellow solid; 96\%; ${ }^{1} \mathrm{H}$ NMR (400 MHz, DMSO- $d_{6}$ ) $\delta 8.10(1 \mathrm{H}, \mathrm{dd}, J 7.5,2.0 \mathrm{~Hz}, \mathrm{H} 5), 7.87(1 \mathrm{H}, \mathrm{dd}, J 7.5$, $2.0 \mathrm{~Hz}, \mathrm{H} 7), 7.74$ (1H, dd, J 7.4, 2.0 Hz, H8), 7.55 (1H, dd, J 7.4, 2.0 Hz, H6), 7.29-7.19 (2H, m, H12, H13); ${ }^{13}$ C NMR 
(100 MHz, DMSO- $d_{6}$ ) $\delta 173.9$ (C4), 155.2 (C9), 152.0 (C2), 135.7 (C11), 135.4 (C3), 134.5 (C47), 129.4 (C13), 125.5 (C12), 125.1 (C5), 124.5 (C6), 121.5 (C10), 118.0 (C8), 115.3 (C14).

\section{2-(Thiophene-2-yl)-4H-chromen-3-yl-sulfonates (4a-4n)}

A solution of compounds (3a-3c) (1 mmol) in anhydrous tetrahydrofuran $(10 \mathrm{~mL})$ and anhydrous chloroform $(10 \mathrm{~mL})$ was added to triethylamine $(4 \mathrm{~mL})$ in a $50 \mathrm{~mL}$ round bottom flask. After the reaction mixture was stirred for $30 \mathrm{~min}$ in an ice bath, sulfonyl chloride $(1.2 \mathrm{mmol})$ was added, and the resulting mixture was refluxed at $80^{\circ} \mathrm{C}$ for 3-4 $\mathrm{h}$ (the end of the reaction was controlled by thin layer chromatography (TLC) using petroleum ether/ethyl acetate $=3: 1$, volume ratio). The resulting solution was then cooled to room temperature and concentrated under vacuum. The residue was poured into water and extracted with ethyl acetate. Afterward, the combined organic layers were washed with water and dried over with anhydrous sodium sulfate, filtered, and concentrated under reduced pressure. Compounds $\mathbf{4 a - 4 n}$ were obtained by column chromatography. Petroleum ether:acetone = 10:1 (v/v).

4a

Yellow solid; 68\%; ${ }^{1} \mathrm{H}$ NMR (400 MHz, DMSO- $d_{6}$ ) $\delta 8.25(1 \mathrm{H}, \mathrm{d}, J 3.9 \mathrm{~Hz}, \mathrm{H} 5), 8.18(1 \mathrm{H}, \mathrm{d}, J 5.0 \mathrm{~Hz}, \mathrm{H} 7), 8.13$ $(1 \mathrm{H}, \mathrm{d}, J 7.9 \mathrm{~Hz}, \mathrm{H} 14), 7.97-7.80$ (2H, m, H6, H12), 7.58 $(1 \mathrm{H}, \mathrm{t}, J 7.5 \mathrm{~Hz}, \mathrm{H} 8), 7.41(1 \mathrm{H}, \mathrm{t}, J 4.5 \mathrm{~Hz}, \mathrm{H} 13), 3.35(3 \mathrm{H}$, s, H18); ${ }^{13} \mathrm{C} \mathrm{NMR}\left(100 \mathrm{MHz}\right.$, DMSO- $\left.d_{6}\right) \delta 172.1$ (C4), 155.2 (C9), 140.7 (C2), 135.5 (C11), 134.8 (C47), 133.7 (C14), 133.2 (C13), 131.1 (C5), 129.3 (C12), 128.6 (6), 126.4 (C3), 125.6 (C10), 118.8 (C8), 41.7 (C18); liquid chromatographyHRMS (LC-HRMS) calcd. for $\left[\mathrm{C}_{14} \mathrm{H}_{10} \mathrm{O}_{5} \mathrm{~S}_{2}\right]^{+}: 322.9970$, found: 323.2770 .

\section{4b}

Yellow solid; $68 \%$; ${ }^{1} \mathrm{H}$ NMR (400 MHz, DMSO- $d_{6}$ ) $\delta$ 8.14-8.07 (3H, m, H5, H2', H6'), 7.87 (1H, td, J 7.4, $1.9 \mathrm{~Hz}, \mathrm{H} 7), 7.77-7.64$ (3H, m, H8, H14, H4'), 7.63-7.51 (3H, m, H6, H3', H5'), 7.43 (1H, dd, J 7.5, 1.7 Hz, H12), $7.33(1 \mathrm{H}, \mathrm{t}, J 7.4 \mathrm{~Hz}, \mathrm{H} 11) ;{ }^{13} \mathrm{C} \mathrm{NMR}\left(100 \mathrm{MHz}, \mathrm{DMSO}-d_{6}\right)$ $\delta$ 174.5 (C4), 155.3 (C9), 149.1 (C2), 138.4 (C11), 137.0 (C1'), 134.5 (C7), 133.4 (C4'), 129.6 (C14), 129.1 (C3', C5'), 128.4 (C2', C6'), 127.5 (C13), 125.9 (C5), 125.3 (C12), 125.0 (C3), 124.5 (C6), 121.9 (C10), 118.0 (C8); LC-HRMS calcd. for $\left[\mathrm{C}_{19} \mathrm{H}_{12} \mathrm{O}_{5} \mathrm{~S}_{2}\right]^{+}: 385.0126$, found: 385.3118 .

4c

Yellow solid; 71\%; ${ }^{1} \mathrm{H}$ NMR (400 MHz, DMSO- $d_{6}$ ) $\delta 8.08(1 \mathrm{H}, \mathrm{dd}, J 5.0,1.2 \mathrm{~Hz}, \mathrm{H} 5), 8.04(1 \mathrm{H}, \mathrm{dd}, J 2.4$, $1.4 \mathrm{~Hz}, \mathrm{H} 7), 8.02(1 \mathrm{H}, \mathrm{t}, J 1.6 \mathrm{~Hz}, \mathrm{H} 14), 7.92-7.85(3 \mathrm{H}$, m, H8, H2', H6'), 7.79 (1H, dd, J 8.5, $1.0 \mathrm{~Hz}, \mathrm{H6}), 7.55$ (1H, ddd, J 8.0, 7.1, 1.1 Hz, H12), 7.46-7.41 (2H, m, H3' H5'), 7.27 (1H, dd, J 5.0, 3.9 Hz, H13), 2.44 (3H, s, H7'); ${ }^{13} \mathrm{C}$ NMR (100 MHz, DMSO- $d_{6}$ ) $\delta 171.3$ (C4), 154.7 (C9), 153.8 (C2), 139.7 (C4'), 137.0 (C11), 135.8 (C1'), 135.3 (C7), 134.2 (C3', C5'), 133.1 (C14), 130.2 (C2', C6'), 129.5 (C13), 128.9 (C5), 128.5 (C12), 126.3 (C3), 125.7 (C6), 123.5 (C10), 118.7 (C8), 21.1 (C7'); LC-HRMS calcd. for $\left[\mathrm{C}_{20} \mathrm{H}_{14} \mathrm{O}_{5} \mathrm{~S}_{2}\right]^{+}:$399.0283, found: 399.028 .

\section{4d}

Yellow solid; 76\%; ${ }^{1} \mathrm{H}$ NMR (400 MHz, DMSO- $d_{6}$ ) $\delta$ 8.09-7.98 (3H, m, H5, H2', H6'), 7.89 (1H, ddd, $J$ 8.7, 7.0, 1.7 Hz, H7), 7.82-7.75 (3H, m, H6, H8, H14), 7.62$7.48(3 \mathrm{H}, \mathrm{m}, \mathrm{H} 12, \mathrm{H} 3$ ', H5') 7.25 (1H, dd, J 5.0, $3.9 \mathrm{~Hz}$, H13), 2.38 (3H, s, H7'); ${ }^{13} \mathrm{C}$ NMR (100 MHz, DMSO- $d_{6}$ ) $\delta 171.3$ (C4), 154.7 (C9), 146.0 (C2), 139.7 (C3'), 135.3 (C11), 134.2 (C1'), 134.1 (C7), 133.1 (C4'), 131.0 (C14), 130.3 (C2'), 130.2 (C6'), 129.0 (C5'), 128.6 (C13), 126.3 (C5), 125.9 (C12), 125.6 (C3), 123.5 (C6), 121.2 (C10), 118.8 (C8), 21.6 (C7'); LC-HRMS calcd. for $\left[\mathrm{C}_{20} \mathrm{H}_{14} \mathrm{O}_{5} \mathrm{~S}_{2}\right]^{+}$: 399.0283, found: 398.1147 .

\section{$4 e$}

Yellow solid; 67\%; ${ }^{1} \mathrm{H}$ NMR (400 MHz, DMSO- $d_{6}$ ) $\delta 8.14$ (3H, ddd, $J$ 9.2, 4.4, $1.3 \mathrm{~Hz}, \mathrm{H} 5, \mathrm{H} 2$ ', H6'), 7.94 $(1 \mathrm{H}, \mathrm{dd}, J$ 8.0, $1.6 \mathrm{~Hz}, \mathrm{H} 7), 7.92-7.77(3 \mathrm{H}, \mathrm{m}, \mathrm{H} 6, \mathrm{H} 8$, H14), 7.74-7.66 (1H, m, H12), 7.60-7.48 (1H, m, H3'), $7.42(1 \mathrm{H}, \mathrm{t}, J 7.9 \mathrm{~Hz}, \mathrm{H} 5$ ') $7.35(1 \mathrm{H}, \mathrm{dd}, J 5.0,3.8 \mathrm{~Hz}$, H13), 2.81 (3H, s, H7'); ${ }^{13} \mathrm{C}$ NMR (100 MHz, DMSO- $d_{6}$ ) d 171.0 (C4), 154.7 (C9), 153.9 (C2), 140.6 (C2'), 138.3 (C11), 136.7 (C4'), 135.4 (C1'), 134.9 (C7), 134.5 (C3'), 133.1 (C14), 133.0 (C6'), 129.4 (C5'), 129.0 (C13), 126.8 (C5), 126.3 (C12), 125.6 (C3), 123.3 (C6), 121.1 (C10), 118.7 (C8), 20.7 (C7'); LC-HRMS calcd. for $\left[\mathrm{C}_{20} \mathrm{H}_{14} \mathrm{O}_{5} \mathrm{~S}_{2}\right]^{+}$: 399.0283, found: 399.0303 .

$4 f$

Purple solid; 70\%; ${ }^{1} \mathrm{H}$ NMR (400 MHz, DMSO- $d_{6}$ ) $\delta 8.10(1 \mathrm{H}, \mathrm{dd}, J 7.5,2.0 \mathrm{~Hz}, \mathrm{H} 5), 7.93-7.83(2 \mathrm{H}, \mathrm{m}, \mathrm{H} 7$, H6'), 7.76-7.67 (2H, m, H14, H4'), 7.67 (1H, dd, J 7.5, $2.1 \mathrm{~Hz}, \mathrm{H} 8), 7.55(1 \mathrm{H}, \mathrm{td}, J 7.5,2.0 \mathrm{~Hz}, \mathrm{H} 6), 7.47(1 \mathrm{H}, \mathrm{dd}$, J 7.4, $1.6 \mathrm{~Hz}, \mathrm{H} 12$ ), 7.41-7.29 (3H, m, HH13, H3', H5'); ${ }^{13} \mathrm{C}$ NMR (100 MHz, DMSO- $\left.d_{6}\right) \delta 171.0(\mathrm{C} 4), 163.5$ (C6'), 161.2 (C9), 154.7 (C2), 154.0 (C11), 138.0 (C1'), 135.0 (C7), 134.7 (C2'), 133.1 (C5'), 131.2 (C14), 130.5 (C13), 129.1 (C5), 126.4 (C12), 125.6 (C3), 125.4 (C6), 123.3 (C10), 118.7 (C8), 118.0 (C3'), 117.8 (C4'); LC-HRMS calcd. for $\left[\mathrm{C}_{19} \mathrm{H}_{11} \mathrm{FO}_{5} \mathrm{~S}_{2}\right]^{+}: 402.0023$, found: 402.0793 . 


\section{$4 g$}

Purple solid; 67\%; ${ }^{1} \mathrm{H}$ NMR (400 MHz, DMSO- $d_{6}$ ) $\delta 8.10(1 \mathrm{H}, \mathrm{dd}, J 5.0,1.2 \mathrm{~Hz}, \mathrm{H} 5), 8.08-8.01(2 \mathrm{H}, \mathrm{m}, \mathrm{H} 14$, $\mathrm{H} 2$ '), 7.88 (3H, m, H7, H8, H2'), $7.81(1 \mathrm{H}, \mathrm{dd}, J$ 8.5, $1.0 \mathrm{~Hz}, \mathrm{H6}), 7.74-7.67$ (2H, m, H12, H4'), 7.55 (1H, ddd, $J$ 8.0, 7.1, 1.1 Hz, H5'), 7.29 (1H, dd, J 5.0, $3.9 \mathrm{~Hz}, \mathrm{H} 13)$; ${ }^{13} \mathrm{C}$ NMR $\left(100 \mathrm{MHz}\right.$, DMSO- $\left.d_{6}\right) \delta 171.2(\mathrm{C} 4), 160.7$ (C3'), 154.8 (C9), 153.9 (C2), 135.4 (C11), 134.5 (C1'), 133.3 (C7), 132.1 (C5'), 130.1 (C14), 126.4 (C13), 125.6 (C6'), 124.9 (C5), 124.9 (C12), 123.4 (C3), 122.6 (C6), 122.4 (C10), 118.8 (C4'), 115.8 (C2'), 115.6 (C8); LC-HRMS calcd. for $\left[\mathrm{C}_{19} \mathrm{H}_{11} \mathrm{FO}_{5} \mathrm{~S}_{2}\right]^{+}: 402.0023$, found: 402.0792 .

\section{4h}

Purple solid; 71\%; ${ }^{1} \mathrm{H}$ NMR (400 MHz, DMSO- $d_{6}$ ) $\delta 8.10(1 \mathrm{H}, \mathrm{dd}, J 7.52 .0 \mathrm{~Hz}, \mathrm{H} 5), 8.03-7.95(2 \mathrm{H}, \mathrm{m}, \mathrm{H} 2$ ', H6'), $7.87(1 \mathrm{H}, \mathrm{td}, J 7.5,2.0 \mathrm{~Hz}, \mathrm{H} 7), 7.74(1 \mathrm{H}, \mathrm{dd}, J 7.5$, $1.6 \mathrm{~Hz}, \mathrm{H} 14), 7.67(1 \mathrm{H}, \mathrm{dd}, J 7.5,2.0 \mathrm{~Hz}, \mathrm{H} 8), 7.55(1 \mathrm{H}$, td, $J 7.5,2.0 \mathrm{~Hz}, \mathrm{H6}), 7.40(1 \mathrm{H}, \mathrm{dd}, J 7.5,1.6 \mathrm{~Hz}, \mathrm{H} 12)$, 7.36-7.27 (3H, m, H13, H3', H5'); ${ }^{13} \mathrm{C}$ NMR $(100 \mathrm{MHz}$, DMSO- $d_{6}$ ) $\delta 174.5$ (C4), 157.0 (C6'), 155.3 (C9), 149.1 (C2), 138.4 (C11), 135.9 (C1'), 134.5 (C7), 130.1 (C2'), 129.6 (C5'), 127.5 (C14), 127.3 (C13), 126.4 (C5), 125.9 (C12), 125.3 (C3), 125.0 (C6), 124.5 (C10), 121.9 (C8), 118.6 (C3'), 118.0 (C4'); LC-HRMS calcd. for $\left[\mathrm{C}_{19} \mathrm{H}_{11} \mathrm{FO}_{5} \mathrm{~S}_{2}\right]^{+}:$402.0023, found: 402.0784 .

\section{$4 \mathbf{i}$}

Yellow solid; 72\%; ${ }^{1} \mathrm{H}$ NMR (400 MHz, DMSO- $d_{6}$ ) $\delta 8.45$ ( $2 \mathrm{H}, \mathrm{dd}, J 8.5,4.1 \mathrm{~Hz}, \mathrm{H} 3$ ', H5'), 8.30 (2H, dd, $J 8.3$, $4.2 \mathrm{~Hz}, \mathrm{H} 2$ ', H6'), 8.12 (2H, dt, J 13.9, 4.4 Hz, H5, H7), 8.02 $(1 \mathrm{H}, \mathrm{t}, J 6.1 \mathrm{~Hz}, \mathrm{H} 14), 7.91(1 \mathrm{H}, \mathrm{td}, J 8.3,7.3,3.5 \mathrm{~Hz}, \mathrm{H} 8)$, 7.87-7.79 (1H, m, H6), 7.61-7.51 (1H, m, H12), $7.32(1 \mathrm{H}$, $\mathrm{p}, J 4.6 \mathrm{~Hz}, \mathrm{H} 13) ;{ }^{13} \mathrm{C}$ NMR (100 MHz, DMSO- $\left.d_{6}\right) \delta 174.5$ (C4), 154.8 (C9), 151.1 (C4'), 149.8(C2), 142.5 (C1'), 135.5 (C11), 134.8 (C7), 133.4 (C14), 130.2 (C2', C6'), 130.0 (C13), 129.2 (C5), 126.5 (C12), 125.6 (C3), 124.9 (C6), 123.3 (C3', C5'), 121.3 (C10), 118.8 (C8); LC-HRMS calcd. for $\left[\mathrm{C}_{19} \mathrm{H}_{11} \mathrm{NO}_{7} \mathrm{~S}_{2}\right]^{+}:$428.9977, found: 429.0497 .

\section{$4 \mathbf{j}$}

Purple solid; 90\%; ${ }^{1} \mathrm{H}$ NMR (400 MHz, DMSO- $d_{6}$ ) $\delta 8.09$ (1H, dd, $J$ 8.6, 5.1 Hz, H5), 8.01 (2H, dd, $J$ 7.8, $2.0 \mathrm{~Hz}, \mathrm{H} 2$ ', H6'), 7.92-7.87 (2H, m, H7, H8), $7.76(1 \mathrm{H}$, d, J 8.5 Hz, H6), 7.51 (3H, dt, J 17.4, $8.2 \mathrm{~Hz}, \mathrm{H} 12, \mathrm{H} 3$ ', H5'), 7.03 (1H, d, J $3.8 \mathrm{~Hz}, \mathrm{H} 13), 2.56$ (3H, s, H17); ${ }^{13} \mathrm{C}$ NMR $\left(100 \mathrm{MHz}\right.$, DMSO- $\left.d_{6}\right) \delta 171.1(\mathrm{C} 4), 162.4\left(\mathrm{C}^{\prime}\right.$ '), 154.69 (C9), 153.8 (C2), 148.9 (C14), 135.3 (C1'), 133.7 (C11), 133.4 (C7), 132.1 (C2'), 132.0 (C6'), 128.3 (C12), 127.5 (C13), 126.3 (C5), 125.6 (C6), 123.4 (C3), 118.7 (C10), 117.1 (C8), 116.9 (C3'), 114.9 (C5'), 15.5 (C17);
LC-HRMS calcd. for $\left[\mathrm{C}_{20} \mathrm{H}_{13} \mathrm{FO}_{5} \mathrm{~S}_{2}\right]^{+}: 417.0188$, found: 417.0189.

\section{4k}

Yellow solid; 85\%; ${ }^{1} \mathrm{H}$ NMR (400 MHz, DMSO- $d_{6}$ ) $\delta$ 8.14-8.06 (2H, m, H5, H7), 7.91 (1H, ddd, $J$ 8.7, 7.1, $1.7 \mathrm{~Hz}, \mathrm{H} 8), 7.79(1 \mathrm{H}, \mathrm{dd}, J 8.5,1.0 \mathrm{~Hz}, \mathrm{H} 6), 7.57(1 \mathrm{H}, \mathrm{ddd}$, $J$ 8.1, 7.1, $1.1 \mathrm{~Hz}, \mathrm{H} 12), 7.13$ (1H, dd, J 3.9, $1.2 \mathrm{~Hz}, \mathrm{H} 13$ ), $3.83(3 \mathrm{H}, \mathrm{s}, \mathrm{H} 18), 2.60(3 \mathrm{H}, \mathrm{d}, J 1.0 \mathrm{~Hz}, \mathrm{H} 17) ;{ }^{13} \mathrm{C}$ NMR $\left(100 \mathrm{MHz}, \mathrm{DMSO}-d_{6}\right) \delta 171.9$ (C4), 154.6 (C9), 153.9 (C14), 149.2 (C2), 135.4 (C11), 133.7 (C7), 128.2 (C12), 127.8 (C13), 126.3 (C5), 125.6 (C6), 124.9 (C10), 123.3 (C3), 118.4 (C8), 41.7 (C18), 15.6 (C17); LC-HRMS calcd. for $\left[\mathrm{C}_{15} \mathrm{H}_{12} \mathrm{O}_{5} \mathrm{~S}_{2}\right]^{+}: 337.0126$, found: 337.0154 .

\section{I}

Yellow solid; 82\%; ${ }^{1} \mathrm{H}$ NMR (400 MHz, DMSO- $d_{6}$ ) $\delta 8.02(1 \mathrm{H}, \mathrm{d}, J 7.9 \mathrm{~Hz}, \mathrm{H} 5), 7.92-7.84(4 \mathrm{H}, \mathrm{m}, \mathrm{H} 7, \mathrm{H} 8, \mathrm{H} 2$ ', H6'), $7.76(1 \mathrm{H}, \mathrm{d}, J 8.4 \mathrm{~Hz}, \mathrm{H} 6), 7.53(1 \mathrm{H}, \mathrm{t}, J 7.5 \mathrm{~Hz}, \mathrm{H} 12)$, $7.44(2 \mathrm{H}, \mathrm{d}, J 8.0 \mathrm{~Hz}, \mathrm{H} 3$ ', H5'), $6.99(1 \mathrm{H}, \mathrm{d}, J 3.7 \mathrm{~Hz}$, H13), 2.54 (3H, s, H17), 2.45 (3H, s, H7'); ${ }^{13} \mathrm{C}$ NMR $\left(100 \mathrm{MHz}, \mathrm{DMSO}-d_{6}\right) \delta 171.2(\mathrm{C} 7), 154.6(\mathrm{C} 2), 148.7$ (C9), 146.0 (C16), 135.3 (C24), 134.2 (C13), 133.6 (C21), 130.1 (C4), 128.6 (C23, C25), 128.4 (C22, C26), 127.8 (C14), 127.6 (C15), 126.3 (C6), 125.9 (C5), 125.6 (C8), 123.5 (C1), 118.7 (C3), 21.6 (C28), 15.5 (C27); LC-HRMS calcd. for $\left[\mathrm{C}_{21} \mathrm{H}_{16} \mathrm{O}_{5} \mathrm{~S}_{2}\right]^{+}: 413.0439$, found: 413.0430 .

\section{$4 m$}

Brown solid; 91\%; ${ }^{1} \mathrm{H}$ NMR (400 MHz, DMSO- $d_{6}$ ) $\delta 8.04(1 \mathrm{H}, \mathrm{d}, J 7.9 \mathrm{~Hz}, \mathrm{H} 5), 7.88(3 \mathrm{H}, \mathrm{t}, J 7.6 \mathrm{~Hz}, \mathrm{H} 7$, H2', H6'), 7.85-7.75 (2H, m, H6, H8), 7.60-7.37 (4H, m, H12, H13, H3', H5'), 2.43 (3H, s, H7'); ${ }^{13} \mathrm{C}$ NMR $\left(100 \mathrm{MHz}, \mathrm{DMSO}-d_{6}\right) \delta 174.3$ (C4), 154.7 (C9), 146.3 (C2), 140.5 (C4'), 135.5 (C1'), 134.1 (C11), 133.6 (C7), 132.1 (C3', C5'), 131.5 (C13), 131.2 (C2', C6'), 130.3 (C5), 128.6 (C6), 126.4 (C3), 125.6 (C12), 123.5 (C10), 120.5 (C8), 118.8 (C14), 21.7 (C7'); LC-HRMS calcd. for $\left[\mathrm{C}_{20} \mathrm{H}_{13} \mathrm{BrO}_{5} \mathrm{~S}_{2}\right]^{+}:$478.9367, found: 478.9360 .

\section{$4 n$}

Brown solid; 99\%; ${ }^{1} \mathrm{H}$ NMR (400 MHz, DMSO- $\left.d_{6}\right)$ $\delta 8.11(1 \mathrm{H}, \mathrm{dd}, J 8.7,5.0 \mathrm{~Hz}, \mathrm{H} 5), 8.03(2 \mathrm{H}, \mathrm{dd}, J 7.9$, $2.1 \mathrm{~Hz}, \mathrm{H} 2$ ', H6'), 7.97-7.83 (2H, m, H7, H8), $7.80(1 \mathrm{H}$, d, J 8.4 Hz, H6), 7.59-7.42 (4H, m, H11, H12, H3', H5'); ${ }^{13} \mathrm{C}$ NMR (100 MHz, DMSO- $d_{6}$ ) $\delta 171.3$ (C4), 154.7 (C4'), 152.4 (C9), 135.5 (C2), 133.8 (C1'), 132.3 (C11), 132.1 (C7), 132.0 (C2', C6'), 131.4 (C13), 131.2 (C5), 126.5 (C6), 125.6 (C12), 123.4 (C3), 120.7 (C10), 118.8 (C8), 117.3 (C3', C5'), 117.0 (C14); LC-HRMS: calcd. for $\left[\mathrm{C}_{19} \mathrm{H}_{10} \mathrm{BrFO}_{5} \mathrm{~S}_{2}\right]^{+}: 482.9117$, found: 482.9100 . 


\section{Antioxidant activity}

The antioxidant activity of compound (4a-4n) was measured spectrophotometrically from the discoloration of ethanolic solution of 2,2-diphenyl-1-picrylhydrazyl (DPPH) at the lambda maximum of its color $(516 \mathrm{~nm})$. Due to the low solubility of the synthesized compound (4a-4n) in ethanol, a stock solution was made for each compound in DMSO so that $1 \mathrm{~mL}$ of each solution was mixed with $9 \mathrm{~mL}$ ethanolic solution of DPPH to form a $10 \mathrm{~mL}$ mixture. These solutions were then kept in the dark for $30 \mathrm{~min}$. Similarly, a $10 \mathrm{~mL}$ solution of $80 \mu \mathrm{M}$ of DPPH was kept in the dark for $30 \mathrm{~min}$, after which the absorbance was measured against the blank sample at $516 \mathrm{~nm} .{ }^{27}$

The other antioxidant activity of compound (4a-4n) was measured spectrophotometrically from the discoloration of ethanolic solution of 2,2'-azino-bis(3-ethylbenzothiazoline6-sulfonic acid) (ABTS) at the lambda maximum of its color $(734 \mathrm{~nm}){ }^{27}$

$\operatorname{SR}(\%)=\left(\frac{\text { control absorbance }- \text { sample absorbance }}{\text { control absorbance }}\right) \times 100$

where SR is the clearance of DPPH or ABTS by the target compound. Ascorbic acid was used as the standard antioxidant for comparison. Data were represented as means \pm standard deviations of triplicate experiments.

\section{Cytotoxic activity}

Human cervical cancer (HeLa), human hepatocellular carcinoma (HepG 2), human lung carcinoma (A549) and L02 cells were obtained from the American Type Culture Collection (ATCC, Rockville, MA, USA) and cultivated (at $37{ }^{\circ} \mathrm{C}$ under an atmosphere containing 5\% $\mathrm{CO}_{2}$ ) in DMEM (Dulbecco's Modified Eagle Medium, Sigma Chemical Company, St. Louis, MO, USA) containing $0.1 \mathrm{mg} \mathrm{mL}^{-1}$ streptomycin, $100 \mathrm{U} \mathrm{mL}^{-1}$ penicillin, and $10 \%$ fetal bovine serum.

Cytotoxicity evaluation using a viability assay was then performed. For cytotoxic assays, the tumor cell lines were suspended in a medium at a concentration of $5 \times 10^{4}$ cells per well in 96-well tissue culture plates, then incubated for $24 \mathrm{~h}$. The tested compounds were then added into 96-well plates (in triplicate) to achieve eight concentrations for each compound $(0,1,2.5,5,10,20,50$ and $100 \mu \mathrm{M})$. After incubation for $44 \mathrm{~h}, 20 \mu \mathrm{L}$ of $5 \mathrm{mg} \mathrm{mL}^{-1}$ of 3-(4,5-dimethylthiazol-2-yl)2,5-diphenyltetrazolium bromide (MTT) were added to each well and incubated for $4 \mathrm{~h}$, and then the culture solution was gently aspirated; $150 \mu \mathrm{L}$ of dimethyl sulfoxide (DMSO) was added to each well to dissolve the methanogenic crystals.
Then, the optical density was measured at $590 \mathrm{~nm}$ with a microplate reader (Molecular Devices, SpectraMax iD5, USA) to determine the number of viable cells, and the percentage of viability was calculated as [(ODt/ODc)] $\times$ $100 \%$, where ODt refers to the mean optical density of wells treated with the tested sample and ODc is the mean optical density of untreated cells. ${ }^{28}$

\section{Results and Discussion}

\section{Chemistry}

Synthetic steps that were adopted to furnish the final 2-(thiophen-2-yl)-4H-chromen-3-yl- sulfonate derivatives (4a-4n) were drawn in Scheme 1. Notably, alcoholic $\mathrm{KOH}$ was used for the synthesis of chalcone (2a-2c) from $o$-hydroxyacetophenone with thiophene-2-carboxaldehyde via Claisen-Schmidt reaction. In addition, $4 H$-chromene (3a-3c) was yielded under acidic conditions by a AlgarFlynn-Oyamada reaction. Arylsulfonyl was selected to introduce the side chain, as it would improve the solubility of the final product in common organic solvents and lead to a better absorption of $4 H$-chromene derivatives by living cells. The synthesized $4 H$-chromene derivatives (4a-4n) were then fully characterized, and their chemical structures, as shown in Scheme 1, were elucidated. Due to the substitution of the arylsulfonyl group, the signal belonging to the 3-OH group disappeared.

\section{Biology}

The results of the pharmacological screening of the newly synthesized 2-(thiophen-2-yl)-4H-chromen-3-ylsulfonate derivatives (4a-4n) as antioxidant molecules tested using 2,2-diphenyl-1-picrylhydrazyl (DPPH) and 2,2'-azino-bis-3-ethylbenzothiazoline-6-sulfonic acid (ABTS), as well as their potential as cytotoxic agents against human cervical cancer (HeLa), human hepatocellular carcinoma (HepG 2), and human lung carcinoma (A549) cell lines. Compounds $\mathbf{4 c - 4 e}$ showed excellent inhibitory effects as summarized in Tables 1 and 2 . In view of the bioassay data obtained in this study, it can be observed that modifying the previously studied $4 H$-chromene conjugates to their sulfonyl precursors, significantly improved the biological efficacy.

Compounds $\mathbf{4 a - 4 n}$ showed significant free radical scavenging ability in the range of $14.73 \pm 0.22$ to $41.57 \pm 0.34 \mu \mathrm{M}$ of $\mathrm{IC}_{50}$ against DPPH and 8.02 \pm 0.41 to $35.68 \pm 0.42 \mu \mathrm{M}$ of $\mathrm{IC}_{50}$ against ABTS. Distinctly, the antioxidant activity of the compounds was significantly affected owing to sulfonyl. 

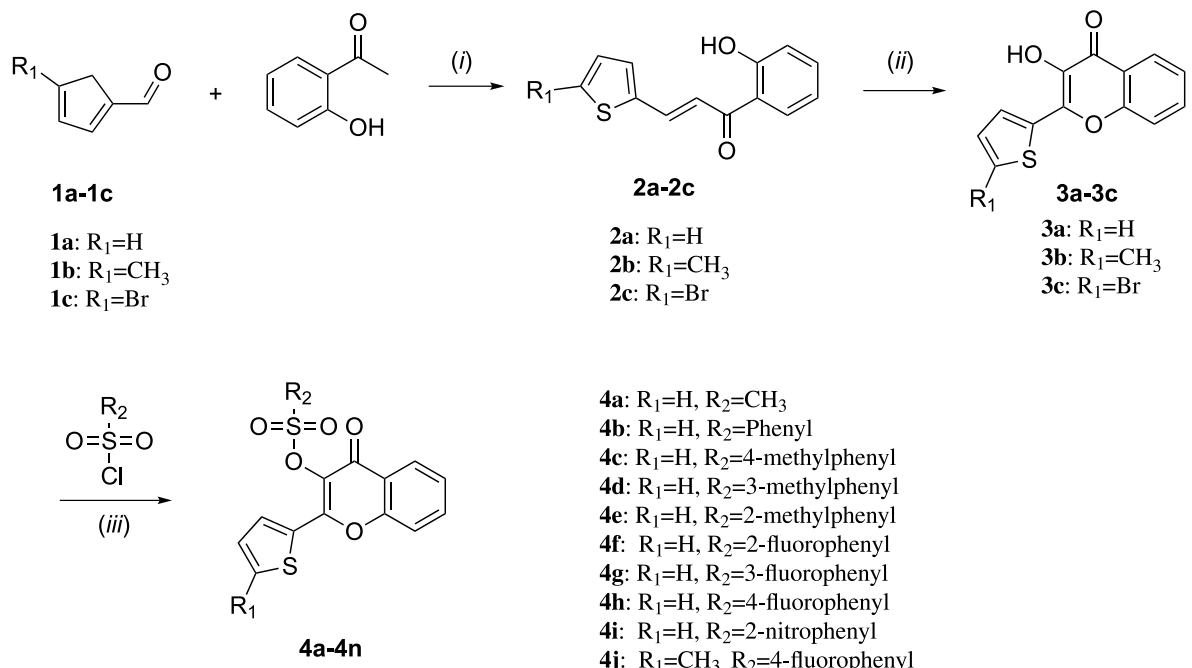

$$
\begin{aligned}
& \text { 4a: } \mathrm{R}_{1}=\mathrm{H}, \mathrm{R}_{2}=\mathrm{CH}_{3} \\
& \text { 4b: } \mathrm{R}_{1}=\mathrm{H}, \mathrm{R}_{2}=\text { Phenyl } \\
& \text { 4c: } \mathrm{R}_{1}=\mathrm{H}, \mathrm{R}_{2}=4 \text {-methylphenyl } \\
& \text { 4d: } \mathrm{R}_{1}=\mathrm{H}, \mathrm{R}_{2}=3 \text {-methylphenyl } \\
& \text { 4e: } \mathrm{R}_{1}=\mathrm{H}, \mathrm{R}_{2}=2 \text {-methylphenyl } \\
& \text { 4f: } \mathrm{R}_{1}=\mathrm{H}, \mathrm{R}_{2}=2 \text {-fluorophenyl } \\
& \text { 4g: } \mathrm{R}_{1}=\mathrm{H}, \mathrm{R}_{2}=3 \text {-fluorophenyl } \\
& \text { 4h: } \mathrm{R}_{1}=\mathrm{H}, \mathrm{R}_{2}=4 \text {-fluorophenyl } \\
& \text { 4i: } \mathrm{R}_{1}=\mathrm{H}, \mathrm{R}_{2}=2 \text {-nitrophenyl } \\
& \text { 4j: } \mathrm{R}_{1}=\mathrm{CH}_{3}, \mathrm{R}_{2}=4 \text {-fluorophenyl } \\
& \text { 4k: } \mathrm{R}_{1}=\mathrm{CH}_{3}, \mathrm{R}_{2}=\mathrm{CH}_{3} \\
& \text { 4l: } \mathrm{R}_{1}=\mathrm{Br}, \mathrm{R}_{2}=\mathrm{CH}_{3} \\
& \text { 4m: } \mathrm{R}_{1}=\mathrm{Br}, \mathrm{R}_{2}=4 \text {-fluorophenyl } \\
& \text { 4n: } \mathrm{R}_{1}=\mathrm{CH}_{3}, \mathrm{R}_{2}=4 \text {-fluorophenyl }
\end{aligned}
$$

Scheme 1. Synthesis of 2-(thiophen-2-yl)-4H-chromen-3-yl-sulfonate derivatives (4a-4n). Reagents and conditions: (i) KOH/MeOH, methanol, reflux; (ii) $\mathrm{KOH} / \mathrm{MeOH}$, methanol, $\mathrm{H}_{2} \mathrm{O}_{2}$, r.t.; (iii) triethylamine, tetrahydrofuran, trichloromethane, reflux.

Table 1. Screening results for DPPH and ABTS radical scavenging activity of 2-(thiophene-2-yl)-4H-chromen-3-yl-sulfonate derivatives (4a-4n)

\begin{tabular}{lcc}
\hline \multirow{2}{*}{ Compound } & \multicolumn{2}{c}{$\mathrm{IC}_{50}{ }^{\mathrm{a}} / \mu \mathrm{M}$} \\
\cline { 2 - 3 } $\mathbf{2 a}$ & $20.51 \pm 0.27$ & $\mathrm{DBPH}$ \\
$\mathbf{3 a}$ & $18.26 \pm 0.16$ & $14.48 \pm 0.34$ \\
$\mathbf{4 a}$ & $28.73 \pm 0.48$ & $26.52 \pm 0.26$ \\
$\mathbf{4 b}$ & $26.49 \pm 0.62$ & $25.57 \pm 0.28$ \\
$\mathbf{4 c}$ & $15.33 \pm 0.47$ & $9.09 \pm 0.11$ \\
$\mathbf{4 d}$ & $14.94 \pm 0.35$ & $8.89 \pm 0.53$ \\
$\mathbf{4 e}$ & $14.73 \pm 0.22$ & $8.02 \pm 0.41$ \\
$\mathbf{4 f}$ & $16.57 \pm 0.28$ & $12.31 \pm 0.09$ \\
$\mathbf{4 g}$ & $17.63 \pm 0.31$ & $13.19 \pm 0.65$ \\
$\mathbf{4 h}$ & $16.99 \pm 0.27$ & $13.48 \pm 0.16$ \\
$\mathbf{4 i}$ & $22.36 \pm 0.14$ & $19.93 \pm 0.35$ \\
$\mathbf{4 j}$ & $37.84 \pm 0.29$ & $30.76 \pm 0.16$ \\
$\mathbf{4 k}$ & $41.57 \pm 0.34$ & $35.68 \pm 0.42$ \\
$\mathbf{4 l}$ & $35.62 \pm 0.17$ & $27.28 \pm 0.15$ \\
$\mathbf{4 m}$ & $35.66 \pm 0.51$ & $31.47 \pm 0.13$ \\
$\mathbf{4 n}$ & $24.33 \pm 0.29$ & $18.37 \pm 0.25$ \\
Ascorbic acid & $12.72 \pm 0.27$ & $5.09 \pm 0.21$ \\
\hline
\end{tabular}

a Data represents the mean values of three independent determinations. $\mathrm{IC}_{50}$ : half maximal inhibitory concentration; DPPH: 2,2-diphenyl1-picrylhydrazyl; ABTS: 2,2'-azino-bis(3-ethylbenzothiazoline-6-sulfonic acid).

Overall, compounds 2a, 3a and 4a-4n expressed a significant level of antiproliferative activity potential in all cell lines. Specifically, compound 3a displayed $10.23 \pm 0.37,14.61 \pm 0.22$ and $15.26 \pm 0.13 \mu \mathrm{M}$ of $\mathrm{IC}_{50}$ against HeLa, HepG 2, and A549 cells. The experimental data were observed to be significantly better than that of $4 \mathrm{H}$-chromen-4-one. It was thoroughly confirmed that the introduction of the thiophene group could dramatically improve cytotoxic activity. Furthermore, compounds $(\mathbf{4} \mathbf{c}-4 \mathbf{e})$ with $-\mathrm{CH}_{3}$ demonstrated excellent activity against HeLa, HepG 2, and A549 cells, even exceeding the control group apigenin. Otherwise, worse cytotoxic activity was represented like compound $\mathbf{4 k}$. Based on the above experimental results, compounds $\mathbf{4 c}-\mathbf{4 e}$ were selected to perform cytotoxicity experiments on L02 cells. The compounds did not exhibit obvious cytotoxicity to L02 cells. Generally, compounds presented variable potencies against HeLa, HepG 2, and A549 cells and had low toxicity to L02 cells, which can be considered to have potential antitumor activity.

\section{Conclusions}

In conclusion, 2-(thiophene-2-yl)-4H-chromen-3-ylsulfonate derivatives $(\mathbf{4 a}-\mathbf{4 n})$ were synthesized, and their structures were elucidated based on ${ }^{1} \mathrm{H}$ NMR, ${ }^{13} \mathrm{C}$ NMR, and HRMS data. The biological evaluation demonstrated advancements against DPPH and ABTS in the antioxidant activity assay. Evidently, compounds with electronwithdrawing groups had better antioxidant activity than compounds with electron-donating groups. Moreover, better antioxidant activity was noted with provisional substitution compounds and parasite substitution compounds. Meanwhile, compounds $\mathbf{4 a - 4 n}$ had their antiproliferative activities evaluated against human hepatocellular carcinoma cells (HepG2), human lung cancer cells (A549), and human cervical carcinoma cells (HeLa). Of these derivatives, 
Table 2. $\mathrm{IC}_{50}$ values of the $4 H$-chromene derivatives against the growth of the HeLa, HepG 2 and A549 in vitro

\begin{tabular}{|c|c|c|c|c|}
\hline \multirow{2}{*}{ Compound } & \multicolumn{4}{|c|}{$\mathrm{IC}_{50}{ }^{\mathrm{a}} / \mu \mathrm{M}$} \\
\hline & $\mathrm{HeLa}$ & HepG 2 & A549 & L02 \\
\hline $2 \mathbf{a}$ & $7.52 \pm 0.51$ & $9.43 \pm 0.23$ & $9.19 \pm 0.48$ & - \\
\hline $3 \mathbf{a}$ & $10.23 \pm 0.37$ & $14.61 \pm 0.22$ & $15.26 \pm 0.13$ & - \\
\hline $4 \mathbf{a}$ & $30.10 \pm 0.25$ & $30.77 \pm 0.31$ & $34.68 \pm 0.49$ & - \\
\hline $4 b$ & $50.39 \pm 0.54$ & $42.83 \pm 0.26$ & $57.56 \pm 0.11$ & - \\
\hline $4 c$ & $4.01 \pm 0.76$ & $4.73 \pm 0.78$ & $4.94 \pm 0.12$ & $83.27 \pm 0.28$ \\
\hline $4 d$ & $3.87 \pm 0.12$ & $5.08 \pm 0.63$ & $4.56 \pm 0.63$ & $107.36 \pm 0.16$ \\
\hline $4 e$ & $3.92 \pm 0.45$ & $6.90 \pm 0.23$ & $6.41 \pm 0.28$ & $95.69 \pm 0.31$ \\
\hline $4 f$ & $18.81 \pm 0.33$ & $15.66 \pm 0.27$ & $18.31 \pm 0.35$ & - \\
\hline $4 \mathrm{~g}$ & $18.56 \pm 0.71$ & $16.67 \pm 0.24$ & $21.38 \pm 0.52$ & - \\
\hline $4 h$ & $47.93 \pm 0.63$ & $66.29 \pm 0.44$ & $57.43 \pm 0.27$ & - \\
\hline $4 i$ & $20.46 \pm 0.65$ & $22.29 \pm 0.16$ & $19.93 \pm 0.41$ & - \\
\hline $4 \mathbf{j}$ & $87.60 \pm 0.27$ & $>100$ & $90.05 \pm 0.16$ & - \\
\hline $4 k$ & $>100$ & $>100$ & $>100$ & - \\
\hline 41 & $50.12 \pm 0.53$ & $52.14 \pm 0.36$ & $40.37 \pm 0.26$ & - \\
\hline $4 m$ & $98.56 \pm 0.14$ & $>100$ & $>100$ & - \\
\hline $4 n$ & $64.45 \pm 0.54$ & $30.19 \pm 0.65$ & $44.28 \pm 0.39$ & - \\
\hline 4H-Chromen-4-one ${ }^{\mathrm{b}}$ & $96.4 \pm 0.56$ & $112.8 \pm 0.36$ & $107.4 \pm 0.47$ & - \\
\hline Apigenin $^{\mathrm{b}}$ & $19.8 \pm 0.98$ & $17.3 \pm 0.22$ & $25.4 \pm 0.58$ & - \\
\hline
\end{tabular}

${ }^{a}$ Data represents the mean values of three independent determinations; ${ }^{\mathrm{b}} 4 \mathrm{H}$-chromen-4-one and apigenin were employed as positive control groups. $\mathrm{IC}_{50}$ : half maximal inhibitory concentration; HeLa: human cervical cancer; HepG 2: human hepatocellular carcinoma; A549: human lung carcinoma; L02: human normal hepatocytes.

compound 3a $\left(\mathrm{IC}_{50}=10.23 \pm 0.37-15.26 \pm 0.13 \mu \mathrm{M}\right)$ displayed excellent activity relative to $4 H$-chromen-4-one $\left(\mathrm{IC}_{50}=96.4 \pm 0.56-112.8 \pm 0.36 \mu \mathrm{M}\right)$. Compounds $4 \mathrm{c}-4 \mathrm{~g}$ $\left(\mathrm{IC}_{50}=3.87 \pm 0.12-21.38 \pm 0.52 \mu \mathrm{M}\right)$ presented excellent activity relative to apigenin against $\mathrm{HeLa}$ $\left(\mathrm{IC}_{50}=19.8 \pm 0.98 \mu \mathrm{M}\right)$, HepG $2\left(\mathrm{IC}_{50}=17.3 \pm 0.22 \mu \mathrm{M}\right)$ and $\mathrm{A} 549\left(\mathrm{IC}_{50}=25.4 \pm 0.58 \mu \mathrm{M}\right)$.

By comparing the results of antioxidant and cytotoxic activity assays of 2-(thiophene-2-yl)- $4 H$-chromen3-yl-sulfonate derivatives (4a-4n), we found that the cytotoxic activity was consistent with the antioxidant damage activity of compounds (4a-4n). 4H-Chromene derivatives inhibit the proliferation of tumor cells by scavenging certain free radicals within the cells. It can be seen that the substitution pattern on the thiophene group at the 2-position and the substituent at the 3-position of the $4 H$-chromene moiety may be considered as a crucial element for cytotoxic activity from the above conformational analysis. Additionally, the incorporation of electron-withdrawing groups at the 3-position facilitates the movement. Accordingly, 2-(thiophen-2-yl)-4H-chromen7-yl-sulfonate derivatives (4a-4n) were illustrated with peak and rationalized structural characteristics. This was found to be uncomplicated, and these derivatives may serve as potentially biologically active molecules.

\section{Supplementary Information}

Supplementary file (containing the NMR and HRMS charts for the synthesized compounds) is available free of charge at https://jbcs.sbq.org.br as PDF file.

\section{Acknowledgments}

We gratefully acknowledge the financial support by fundamental scientific research business expenses of colleges and universities in Heilongjiang Province (2020-KYYWF-0023) and Qiqihar Institute of Medical Sciences Project (QMSI2020M-09).

\section{References}

1. Bu, M.; Cao, T. T.; Li, H. X.; Guo, M. Z.; Zhou, Y.; Zhang, N.; Hu, L. M.; Bioorg. Med. Chem. Lett. 2017, 27, 3856.

2. Thomas, N.; Zachariah, S. M.; Int. J. Pharm. Sci. Rev. Res. 2013, 22, 50 .

3. Magedov, I. V.; Manpadi, M. N.; Evdokimov, M.; Elias, E. M.; Bioorg. Med. Chem. Lett. 2007, 17, 3872.

4. Singh, O. M.; Devi, N. S.; Thokchom, D. S.; Sharma, G. J.; Eur. J. Med. Chem. 2010, 45, 2250.

5. Vukovic, N.; Sukdolak, S.; Solujic, S.; Niciforovic, N.; Food Chem. 2010, 120, 1011. 
6. Winter, E.; Lecerf-Schmidt, F.; Gozzi, G.; Peres, B.; Lightbody, M.; Gauthier, C.; Ozvegy-Laczka, C.; Szakacs, G.; Sarkadi, B.; Creczynski-Pasa, T. B.; Boumendjel, A.; Di Pietro, A.; J. Med. Chem. 2013, 56, 9849.

7. Kandioller, W.; Kubanik, M.; Bytzek, A. K.; Jakupec, M. A.; Roller, A.; Keppler, B. A.; Hartinger, C. G.; Tetrahedron 2015, $71,8953$.

8. Liu, Y. H.; Xia, B.; Lan, J. J.; Hu, S. C.; Huang, L.; Pan, W. D.; Molecules 2017, 22, 1923.

9. Majid, Z.; Seyed, A. R.; Hossein, H.; Biomed. Pharmacother. 2017, 92, 998.

10. Cichon, N.; Saluk-Bijak, J.; Gorniak, L.; Przyslo, L.; Bijak, M.; Antioxidants 2020, 9, 1035.

11. Parmenter, B. H.; Croft, K. D.; Hodgson, J. M.; Dalgaard, F.; Bondonno, C. P.; Lewis, J. R.; Cassidy, A.; Scalbert, A.; Bondonno, N. P.; Food Funct. 2020, 11, 6777.

12. Murray, I. A.; Patterson, D. A.; Perdew, G. H.; Nat. Rev. Cancer 2014, 14, 801

13. Ottaviani, A.; Iacovelli, F.; Idili, A.; Falconi, M.; Ricci, F.; Desideri, A.; Nucleic Acids Res. 2018, 46, 9951.

14. Carr, M.; Knox, A. J. S.; Nevin, D. K.; O’Boyle, N.; Wang, S.; Egan, B.; McCabe, T.; Twamley, B.; Zisterer, D. M.; Lloyd, G. L.; Meegan, M. J.; Bioorg. Med. Chem. 2020, 28, 115261.

15. Jiang, X. Y.; Guo, J. N.; Lv, Y. J.; Yao, C. S.; Bioorg. Med. Chem. 2020, 28, 115550.

16. Hikita, K.; Saigusa, S.; Takeuchi, Y.; Matsuyama, H.; Nagaia, R.; Kato, K.; Murata, T.; Tanaka, H.; Wagh, Y. S.; Asao, N.; Kaneda, N.; Bioorg. Med. Chem. 2020, 28, 115490.

17. Swain, B.; Angeli, A.; Singh, p.; Supuran, C. T.; Arifuddin, M.; Bioorg. Med. Chem. 2020, 28, 115586.
18. Yang, G.; Cheng, C.; Xu, G. B.; Tang, L.; Chua, K. L.; Yang, Y. Y.; Bioorg. Med. Chem. 2020, 28, 115606.

19. Ferreira R. J.; Gajdács, M.; Kincses, A.; Spengler, G.; dos Santos, D. J. V. A.; Ferreira, M.-J. U.; Bioorg. Med. Chem. 2020, 28, 115798.

20. Oh, S. K.; Lee, I. O.; Lim, B. G.; Jeong, H.; Int. J. Med. Sci. 2019, 16, 1439.

21. Ruiz, A.; Gutierrez, I. H.; Mardones, C.; Vergara, C.; Herlitz, E.; Vega, M.; Dorau, C.; Winterhalter, P.; Baer, D.; J. Agric. Food Chem. 2010, 58, 6081.

22. Heaney, M. L.; Gardner, J. R.; Karasavvas, N.; Golde, D. W.; Scheinberg, D. A.; Smith, E. A.; O`Connor, O. A.; Cancer Res. 2008, 68, 8031.

23. Kok, Y. Y.; Mooi, L. Y.; Ahmad, K.; Sukari, M. A.; Mat, N.; Rahmani, M.; Ali, A. M.; Molecules 2012, 17, 4651.

24. El-Deeb, I. M.; Bayoumi, S. M.; El-Sherbeny, M. A.; AbdelAziz, A. A. M.; Eur. J. Med. Chem. 2010, 45, 2516.

25. Abdel-Aziz, A. A. M.; El-Azab, A. S.; Ekinci, D.; Şentürk, M.; Supuran, C. T.; J. Enzyme Inhib. Med. Chem. 2015, 30, 81.

26. Li, X.; Li, Y.; Xu, W.; Bioorg. Med. Chem. 2006, 14, 1287.

27. Mughal, E. U.; Sadiq, A.; Murtaza, S.; Rafique, H.; Zafar, N. M.; Riaz, T.; Khan, B. A.; Hameed, A.; Khan, K. M.; Bioorg. Med. Chem. 2017, 25, 100.

28. Al Zahrani, N. A.; El-Shishtawy, R. M.; Elaasser, M. M.; Asiri, A. M.; Molecules 2020, 25, 4566.

Submitted: February 17, 2021

Published online: May 7, 2021 